\title{
Energy harvesting from nanofibers of hybrid organic ferroelectric dabcoHReO 4
}

D. Isakov, E. de Matos Gomes, B. Almeida, A. L. Kholkin, P. Zelenovskiy, M. Neradovskiy, and V. Ya. Shur

Citation: Appl. Phys. Lett. 104, 032907 (2014); doi: 10.1063/1.4862437

View online: http://dx.doi.org/10.1063/1.4862437

View Table of Contents: http://aip.scitation.org/toc/apl/104/3

Published by the American Institute of Physics

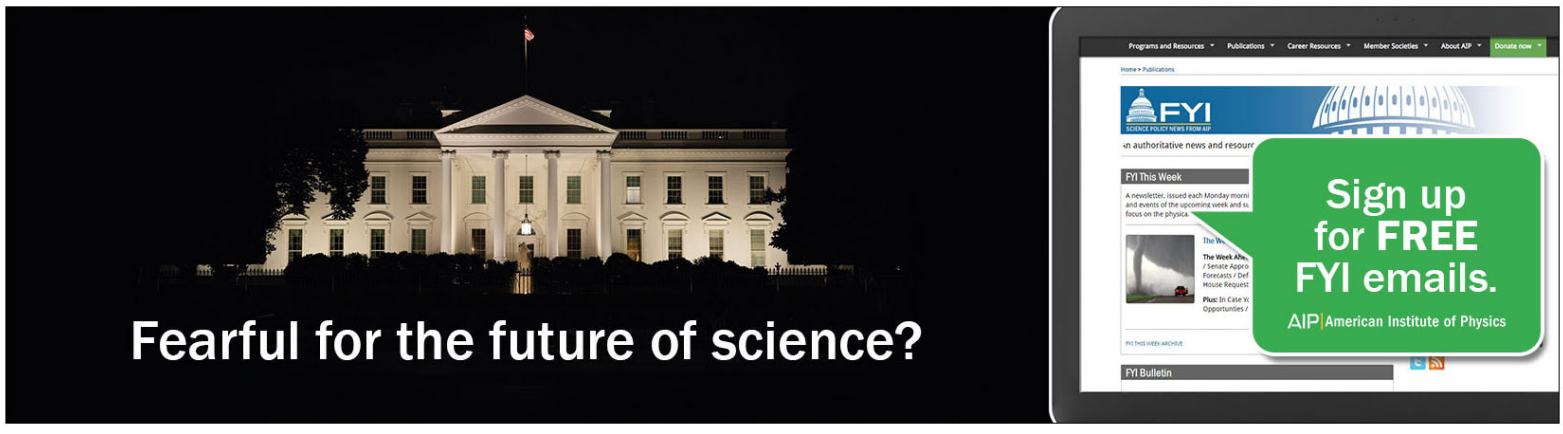




\title{
Energy harvesting from nanofibers of hybrid organic ferroelectric dabcoHReO$_{4}$
}

\author{
D. Isakov, ${ }^{1}$ E. de Matos Gomes, ${ }^{1}$ B. Almeida, ${ }^{1}$ A. L. Kholkin, ${ }^{2,3}$ P. Zelenovskiy, ${ }^{3}$ \\ M. Neradovskiy, ${ }^{3}$ and V. Ya. Shur ${ }^{3}$ \\ ${ }^{1}$ Center of Physics, University of Minho, Campus de Gualtar, 4710057 Braga, Portugal \\ ${ }^{2}$ Department of Materials and Ceramics Engineering (CICECO), University of Aveiro, 3810193 Aveiro, \\ Portugal \\ ${ }^{3}$ Ural Federal University, Lenin Ave. 51, Ekaterinburg 620083, Russia
}

(Received 12 November 2013; accepted 5 January 2014; published online 23 January 2014)

\begin{abstract}
We report excellent piezoelectric and pyroelectric properties of electrospun nanofibers based on the hybrid ferroelectric 1,4-diazabicyclo[2.2.2] octane perrhenate (dabcoHReO 4 ). Ferroelectric nanoparticles are embedded into the fibers being naturally aligned with the major polarization component along the fiber axis. A flexible piezoelectric nanogenerator consisting of an aligned array of dabcoHReO $\mathrm{H}_{4}$ fibers provides a voltage above $100 \mathrm{mV}$ under a moderate strain level. The pyroelectric coefficient in as-electrospun dabcoHReO$_{4}$ fiber mat is similar to that in poled polyvinylidene difluoride nanofibers. The results show that the nanofibers based on $\mathrm{dabcoHReO}_{4}$ have a great potential for pyroelectric and piezoelectric autonomous energy harvesting with natural advantages such as biocompatibility, flexibility, low cost, and easy fabrication. (C) 2014 AIP Publishing LLC. [http://dx.doi.org/10.1063/1.4862437]
\end{abstract}

Flexible electronics have recently attracted great interest due to its potential applications in portable devices such as nanogenerators for energy conversion, storage devices, photodetectors, transistors, and solar cells. ${ }^{1-3}$ The development of smart textiles is also an emerging research field having promising multiple impacts on various technological applications including wearable electronics, implantable physiological sensing and monitoring, signal and power pathways, large surface lighting or heating, and electromagnetic shielding or textile antennas. ${ }^{4-6}$ The common approach to design functional fabric for wearable devices consists of attaching conventional off-shelf micro-electronic components such as transducers, light emitting diodes, and microcomputers to the clothes. ${ }^{7,8}$ Such devices must be powered by external batteries which limit their mobility and increase the system size.

On the other hand, functionalities such as piezoelectricity, ${ }^{9}$ light wave conversion, ${ }^{10}$ or light emission ${ }^{11}$ can be intrinsic to nonwoven textile fibers produced by electrospinning. These works have demonstrated that the electrospinning technique has the potential to be reliable and cost effective nanomanufacturing process in the field of flexible electronics. The unique characteristics of electrospun nanofibers such as their high surface-to-volume ratio, biochemical flexibility, and the possibility of obtaining extremely long lengths are critically important for a variety of technological applications. ${ }^{12}$ Besides, the electrospinning is one of the few nanotechnology methods, which is feasible to scale-up to an industrial level. $^{13}$

An important feature of nanofibers produced by the electrospinning technique is the strong shear and electrostatic forces exerted during their formation which causes the alignment of dopants, molecules, and chains within the principal fiber axis. ${ }^{14,15}$ This feature is especially attractive for organic polar materials, since promoting such an internal molecular orientation (self-assembly) of the organic molecules can result in useful functionalities, e.g. strong polarized emission $^{16}$ or enhanced polar properties. ${ }^{17}$ This highly anisotropic alignment of active components is expected to bring in novel possibilities in the view of potential applications. Recent success in the electrospinning of peptides and polymer-free materials is very encouraging for fabrication of a variety of organic nanofibers with strong $\pi \pi$ or H-bonding interactions that can serve as a molecular scaffolding for the stabilization of continuous fiber-like structures. ${ }^{18,19}$ Additionally, the interest in electrospun nanofibers reveals a big potential of this low-cost nanomanufacturing process for the development of fiber-based energy harvesting elements based on piezoelectric effect. ${ }^{20-22}$

In this work, we report a successful fabrication of nanofibers based on 1,4-diazabicyclo[2.2.2]-octane perrhenate $\left(\right.$ dabcoHReO$\left._{4}\right)$ and demonstrate its usefulness for energy microharvesting and thermal sensing. $\mathrm{DabcoHReO}_{4}$ is a molecular analogue of the well-known inorganic ferroelectric and nonlinear optical material potassium dihydrogen phosphate (KDP) that crystallizes in a monoclinic polar structure belonging to the space group $\mathrm{Cm}$. It is built of $\mathrm{NH} \cdots \mathrm{N}$ hydrogen-bonded dabcoH cations aligned along the [001] polar axis. These $\mathrm{NH} \cdots \mathrm{N}$ hydrogen bond networks directly contribute to the spontaneous polarization along the proton-donor to proton-acceptor direction. ${ }^{23}$ This crystal is a room temperature ferroelectric salt with a spontaneous polarization $P_{s}$ of $17 \mu \mathrm{C} / \mathrm{cm}^{2}$ along the polar axis, being the largest among water-soluble organic ferroelectrics.

Continuous uniform dabcoHReO $\mathrm{H}_{4}$ fibers with an average diameter of about $400 \mathrm{~nm}$ were produced by a conventional electrospinning process, using a precursor aqueous solutions obtained by mixing dissolved dabcoHReO ${ }_{4}$ crystals and poly(vinyl alcohol) (PVA) in a 1:1 wt. \% ratio. The precursor solution was loaded into a syringe which was pumped at $1 \mathrm{ml} / \mathrm{h}$ flow rate with $1012 \mathrm{kV}$ applied between the needle 
and the ground collector. The collector was kept at distance of $12 \mathrm{~cm}$ from the anode needle.

The crystalline structure of dabcoHReO${ }_{4}$ embedded in PVA nanofibers was accessed by $\Theta 2 \Theta$ X-ray diffraction (XRD) scans recorded on Bruker D8 Discover X-ray diffractometer. Nanocrystals of dabcoHReO${ }_{4}$ and the polymer nanofiber matrix form separate phases without the formation of any new complex. The relative intensities of the observed diffraction peaks in the $\mathrm{X}$-ray diffraction pattern (Figure 1(a)) deviate from those predicted for a polycrystalline sample. The most intensive Bragg reflection is (200) followed by (110) in the fibers while in bulk the most intense reflections are (110), (11-1), (111), and (200) in order of decreasing intensity. The reflections of bulk PVA (occurring at $2 \Theta=16.0^{\circ}, 19.4^{\circ}, 20.1^{\circ}$, and $22.7^{\circ}$ ) are not observed, leading to the conclusion that PVA polymer inside the fibers is amorphous. $^{24}$

The XRD result shows that the nanocrystals of dabcoHReO ${ }_{4}$ embedded into the fibers are oriented in such a way that one-dimensional $\mathrm{NH} \cdots \mathrm{N}$ hydrogen bonded chains are parallel to the fibers mat plane. As was mentioned above, the alignment of nanoparticles embedded into the electrospun fibers with their dipole moment directed along the fiber is a common feature of nanofibers produced by the electrospinning. It is important to note that the $\mathrm{NH} \cdots \mathrm{N}$ hydrogen bond network directly contributes to the spontaneous polarization along the proton-donor to proton-acceptor direction. ${ }^{23}$

Raman spectroscopy was employed with the objective of determining the microscopic orientations of dabcoHReO${ }_{4}$ nanocrystals inside the fibers. Raman measurements were performed using a confocal Raman microscope WiTec alpha300AR (WiTec $\mathrm{GmbH}$, Germany) with a $3.19 \mathrm{~cm}^{-1}$ spectral resolution and $200 \mathrm{~nm}$ and $500 \mathrm{~nm}$ of lateral and vertical optical resolutions, respectively. The spectra were
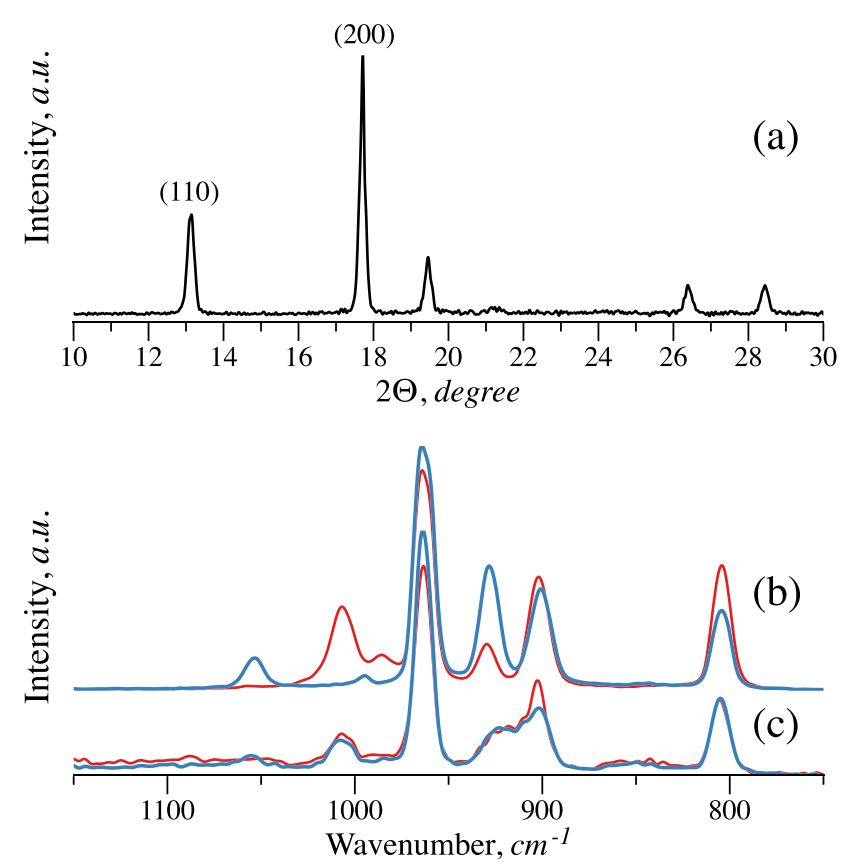

FIG. 1. (a) $\mathrm{X}$ ray diffraction pattern from dabcoHReO $\mathrm{O}_{4}$ fibers mat; (b) and (c) polarized Raman spectra of dabcoHReO$_{4}$ crystal and aligned array of nanofibers, correspondingly. The vertical $(E \| Z)$ and horizontal $(E \perp Z)$ ori entations of the polarization of incident light is assigned by red and blue curves, correspondingly. measured in a backscattering configuration with the laser polarization oriented along $(E \| Z)$ and perpendicular $(E \perp Z)$ to the aligned bundle of dabcoHReO $\mathrm{O}_{4}$ fibers (Figure 1(c)). The dabcoHReO ${ }_{4}$ single crystal oriented with its $Z$ polar axis either parallel or perpendicular to the polarization has been used as a reference (Figure 1(b)).

Several Raman lines have been chosen for the assessment of the orientation of dabco molecules inside the fiber. The bands at $1007 \mathrm{~cm}^{1}$ and $930 \mathrm{~cm}^{1}$ correspond, to symmetric and antisymmetric stretching vibrations of $\mathrm{C} \mathrm{C}$ bonds respectively and characterize the orientation of the dipole moment of the dabco molecule. ${ }^{25,26}$ The presence of a $1007 \mathrm{~cm}{ }^{1}$ line in the spectrum of nanofibers indicates that dabco molecules are mainly oriented in the plane of the mat, whereas its angular independence shows the deviation of molecules from the fiber's axis at a certain angle. An average value of this angle was found from analysis of intensity of several $\mathrm{A}_{1}$ Raman bands including lines at 803 and $1007 \mathrm{~cm}{ }^{1}$. Considering the Raman polarizability tensor, ${ }^{27}$ we found that dabco molecules are in the $b c$-plane and are rotated along $Z$-axis with average angle of $23^{\circ}$. These results are in line with the XRD data, which show that the most intense peak corresponds to (200) plane which is parallel to $b c$-plane.

The ferroelectric and piezoelectric properties of the dabcoHReO ${ }_{4}$ nanofibers have been evaluated by the piezoresponse force microscopy (PFM) technique. ${ }^{28}$ PFM was performed using a NTEGRA Aura (NT-MDT) instrument where ac and dc voltages were applied between the counter electrode and the conductive tip (frequency of ac voltage 50 $\mathrm{kHz}$ and $1 \mathrm{~V}$ peak-to-peak amplitude). During scanning, the vertical and lateral signals from the photodiode were amplified by the lock-in amplifier (SR830A) and imaged using auxiliary channels of the microscope.

Figure 2(b) represents the PFM image obtained in vertical (out-of-plane) mode where the local electric field is

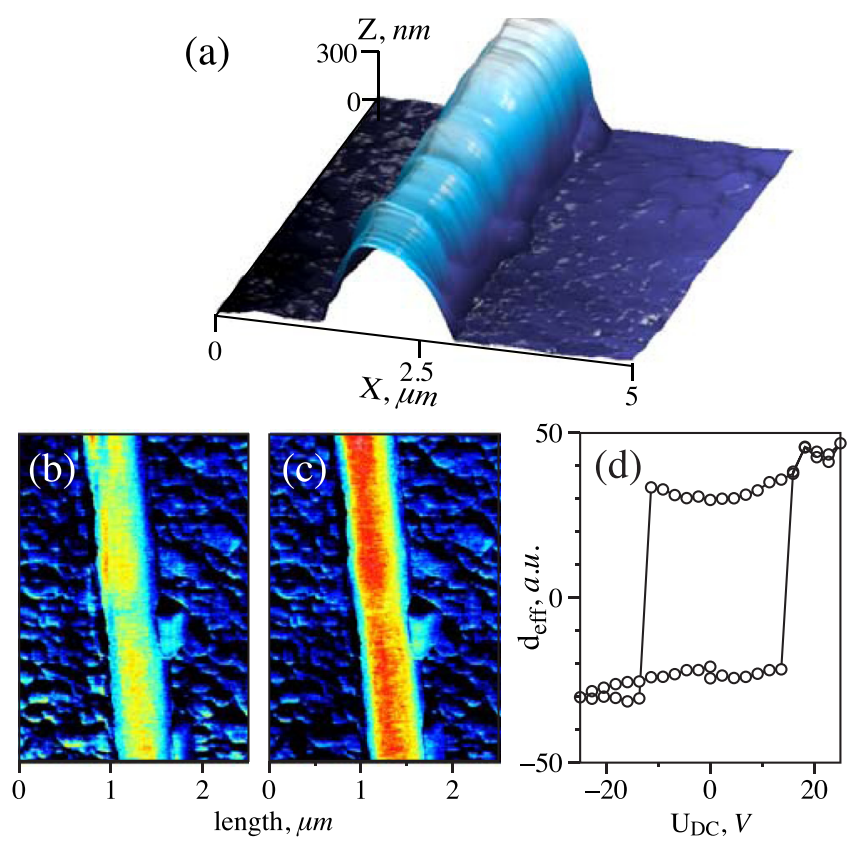

FIG. 2. (a) Morphology of the dabcoHReO $\mathrm{O}_{4}$ individual fiber obtained by AFM; (b) vertical and (c) lateral PFM images of dabcoHReO ${ }_{4}$ nanofibers; (d) piezoelectric response hysteresis loop. 
applied normal to the fiber axis. Vertical PFM enables to distinguish polarization domains with the out-of-plane component of the polarization (that are normal to the fiber axis), while lateral PFM determines the polarization component parallel (in-plane) to the sample surface. As seen from Figures 2(b) and 2(c), the piezoelectric response is stronger along the fiber axis. The detailed examination of in-plane PFM images does not reveal any visible domain structure, indicating that dabcoHReO ${ }_{4}$ nanoparticles are homogeneously dispersed within the fiber.

The piezoelectric effect in dabcoHReO $\mathrm{O}_{4}$ (point group $m$ ) is characterized by a third rank tensor with ten independent coefficients. Assuming that the polar axis $\mathrm{Z}$ in dabcoHReO${ }_{4}$ crystals embedded into the fiber has preferential orientation mainly in the plane of the fiber direction (while $a c$ and $b c$ planes have random orientation), $d_{11}$ and $d_{12}$ components of the piezoelectric tensor may contribute to the effective vertical PFM response $\left(d_{\mathrm{eff}}\right)$, while $d_{13}, d_{15}$, and $d_{24}$ coefficients influence the observed transversal PFM response. We assume that the conglomerates of differently oriented dabcoHReO ${ }_{4}$ nanoparticles are seen as an uniform contrast in both out-of-plane and in-plane piezoresponse imaging.

The local domain switching under an external electric field was studied by means of piezoresponse hysteresis loop acquisition. In these measurements, the tip was fixed on a fiber surface in the central part and a sequence of dc bias voltage pulses with amplitude cycled from -25 to $+25 \mathrm{~V}$ was applied between tip and the counter electrode. The observed out-of-plane hysteresis loop directly proves the switchability of polarization in dabcoHReO $\mathrm{H}_{4}$ nanofibers (Figure 2(d)). The corresponding effective piezoelectric coefficient (remanent value) was estimated to be about $d_{\mathrm{eff}} \approx 20 \mathrm{pm} / \mathrm{V}$. This value is comparable to the piezoelectric coefficient of a poled poly(vinydelene fluoride) film, a classical ferroelectric polymer frequently used for fibers fabrication. $^{29}$

As we observed a pronounced piezoelectric effect in dabcoHReO4 fibers, we explored its suitability for energy harvesting applications. Modern electronics does require a power of the order of less than a microwatt, and thus electrospun fibers may be used to supply energy for miniaturized systems. ${ }^{30}$ In order to demonstrate the power generation ability of the dabcoHReO${ }_{4}$ nanofibers, the piezoelectric response was measured under a periodic bending of the fibers directly electrospun on a flexible polyimide substrate with a sputtered comb-shaped array of 6 pairs of gold electrode plates with outward area $30 \times 10 \mathrm{~mm}^{2}$ (as described elsewhere ${ }^{20,21}$ ). The substrate stripe was bend using a vibration generator with a periodic bending oscillation in the range of $1-5 \mathrm{~Hz}$. The periodic bending results in a strong piezoelectric voltage response with a periodic oscillation between positive and negative output peaks, corresponding to the bending and release cycles (Figure 3). The raw output signal reaches a maximum (peak) value of about $120 \mathrm{mV}$ at $5 \mathrm{~Hz}$.

The energy conversion efficiency of the dabcoHReO $\mathrm{H}_{4}$ piezoelectric device can be considered equal to the effective electromechanical coupling factor $\eta=W_{e} / W_{m}$, where $W_{e}$ is the electrical output energy, and $W_{m}$ is the mechanical input energy. The $W_{m}$ can be calculated as $W_{m}=\int F d x$, where $F$ is the applied force to the stripe oscillator which is

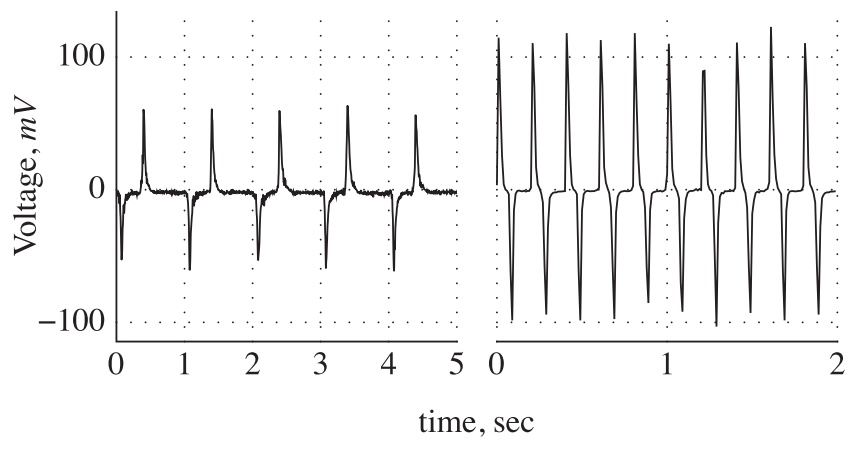

FIG. 3. Voltage output of the fabricated nanofiber mat under $1 \mathrm{~Hz}$ (left) and $5 \mathrm{~Hz}$ (right) repeated compressive impacts. Nanofiber mat thickness of $100 \mu \mathrm{m}$, working area of $1 \mathrm{~cm}^{2}$.

proportional to the distance traveled by its support $x=x_{\max } \sin (\omega t)$. For small deviation of the substrate stripe with dabcoHReO ${ }_{4}$ nanofibers, the calculated conversion efficiency is about $14.8 \%$ at $5 \mathrm{~Hz}$. The nanofibers of dabcoHReO ${ }_{4}$ are capable of producing an output power of about $1.5 \mathrm{nW}$ that might be already suitable to power CMOS or quartz oscillator microdevices.

Next, we demonstrate the direct heat conversion into electricity by means of a pyroelectric effect in a dabcoHReO ${ }_{4}$ nanofiber array. Pyroelectricity results from the temperature dependence of the spontaneous polarization. By changing the temperature, an electric field originated from changes in intrinsic dipoles is compensated by the surface layer of free charges. The rate of change of spontaneous polarization $\frac{d P_{s}}{d T}$ determines the pyroelectric coefficient. The change in the polarization can be detected as a pyroelectric current $I=A \cdot d P_{s} / d T \cdot d T / d t$, where $A$ is electrode area and $d T / d t$ is the rate of temperature change, measured in a capacitor geometry under short-circuit conditions. ${ }^{31}$

Figure 4 presents the variation of a pyroelectric current obtained from $1 \mathrm{~cm}^{2}$ nanofibers mat of about $100 \mu \mathrm{m}$

(a)
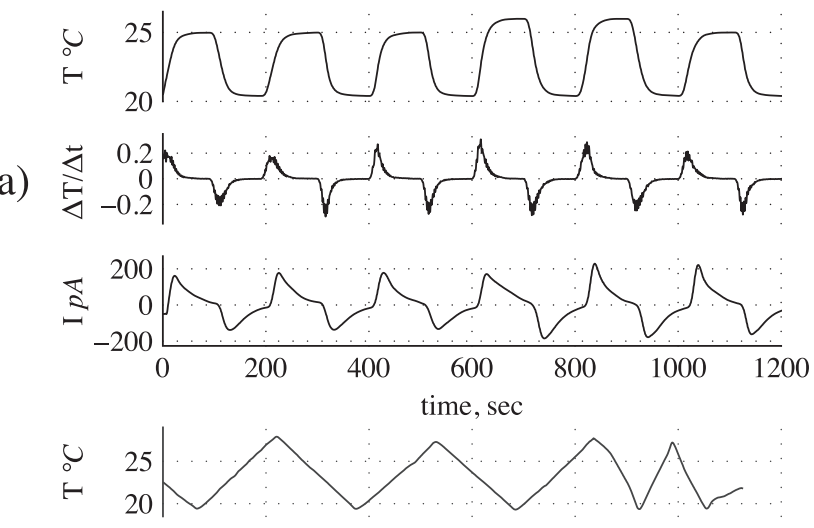

(b)

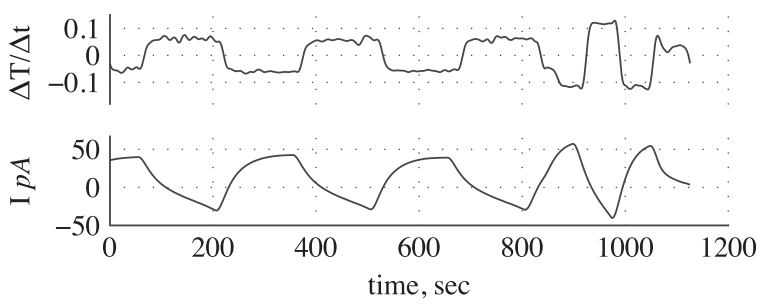

FIG. 4. The temperature cyclic change, derivative and output pyroelectric current obtained in dabcoHReO $\mathrm{H}_{4}$ nanofiber mat at pulsed (a) and ramp (b) temperature changes. 
thickness sandwiched between two electrodes and measured by varying the temperature with different amplitude in the vicinity of room temperature. The current measurements were carried out by using a Keithley 617 electrometer, with the fibers under a constant stress to avoid the piezoelectric contribution. The pyroelectric response is nearly symmetrical with respect to heating/cooling and $I(t)$ follows the shape of the derivative $\frac{d T}{d t}(t)$. The maximum current peak was $200 \mathrm{pA}$ at $\Delta T=6 \mathrm{~K}$. To evaluate the effective pyroelectric coefficient, a temperature ramp with a constant heating and cooling rate was used (Figure 4(b)). As expected, the current increases with increasing temperature. The corresponding pyroelectric coefficient of the as-spun dacboHReO $\mathrm{O}_{4}$ nanofibers has been found to be about $8.5 \mu \mathrm{C} / \mathrm{m}^{2} \mathrm{~K}$. This value is comparable with the reported pyroelectric coefficient of a poled PVDF film ${ }^{32}$ and $\mathrm{KNbO}_{3}$ nanowire-polymer composite. ${ }^{33}$ In spite of the lower pyroelectric coefficient measured for dabcoHReO ${ }_{4}$ nanofibers when compared to bulk crystals (our measurements in single crystal of dabcoHReO $\mathrm{H}_{4}$ grown from aqueous solution of the perrhenate acid and dabco, reveal the pyroelectric coefficient $p_{x}$ of about $46 \mu \mathrm{C} / \mathrm{m}^{2} \mathrm{~K}$ ), the fiber mat has a great advantage over single crystals because it is easier to incorporate into electrical devices; it has lower heat capacity and presents a pyroelectric coefficient that is similar to that of the best current alternative candidates. Moreover, the bulk crystals are very expensive to grow due to their very high solubility in water.

As follows from the structural measurements, we may conclude that a higher percentage of dabcoHReO $\mathrm{O}_{4}$ nanocrystals oriented such as one-dimensional $\mathrm{NH} \cdots \mathrm{N}$ hydrogen bonded chains are parallel to the fibers mat, and the spontaneous polarization has a higher value when measured along the fiber plane. According to point group $m$, the main contribution to the pyroelectric current response must be assigned to the transversal component $P_{S_{X}}$. From the results of Szafrański et al. ${ }^{23}$ in single crystal of dabcoHReO${ }_{4}$, the spontaneous polarization $P_{s_{X}}=3.6 \mu \mathrm{C} / \mathrm{cm}^{2}$ is five times smaller than $P_{s_{Z}}$. According to the electrode position used, the pyroelectric coefficient measured in the fibers has only the contribution of spontaneous polarization perpendicular to the fiber axis.

To summarize, fully flexible devices of organic ferroelectric dabcoHReO $\mathrm{H}_{4}$ nanofibers produced by the low-cost electrospinning process have been fabricated and characterized. The piezoelectric harvesting capability was demonstrated. Both piezoelectric and pyroelectric coefficients are sufficiently high and open up a pathway to incorporating them in smart textile to serve as a harvester of sub-microwatt energy. Future applications are also foreseen in the area of MEMS/NEMS where actuation is required at the nanoscale. Additionally, the possibility to produce flexible self-powered systems would greatly increase the range of potential applications of electrospun nanofibers.

This work was financially supported by the European Regional Development Fund (ERDF) through Programa Operacional Factores de Competitividade (COMPETE: FCOMP-01-0124-FEDER-014628, FCOMP-01-0124FEDER-009457) and the Portugal Fundação para a Ciência e Tecnologia (PTDC/CTMNAN/114269/2009) and was partly supported by the FCT Grant Pest-C/CTM/LA0011/013 and by RFBR (Grant No. 13-02-90925). The equipment of the Ural Center for shared use "Modern Nanotechnology" Institute of Natural Sciences of Ural Federal University has been used. We are indebted to I. Bdikin for help with PFM measurements.

${ }^{1}$ J. M. Wu, C. Xu, Y. Zhang, Y. Yang, Y. Zhou, and Z. L. Wang, Adv. Mater. 24, 6094 (2012).

${ }^{2}$ X. Wang, W. Song, B. Liu, G. Chen, D. Chen, C. Zhou, and G. Shen, Adv. Funct. Mater. 23, 1202 (2013).

${ }^{3}$ W. Kylberg, F. Araujo de Castro, P. Chabrecek, U. Sonderegger, B. T. T. Chu, F. Nuesch, and R. Hany, Adv. Mater. 23, 1015 (2011).

${ }^{4}$ M. Park, J. Im, M. Shin, Y. Min, J. Park, H. Cho, S. Park, M. B. Shim, S. Jeon, D. Y. Chung, J. Bae, J. Park, U. Jeong, and K. Kim, Nat. Nanotechnol. 7, 803 (2012).

${ }^{5}$ D. Zou, Z. Lv, X. Cai, and S. Hou, Nano Energy 1, 273 (2012).

${ }^{6}$ B. S. Shim, W. Chen, C. Doty, C. L. Xu, and N. A. Kotov, Nano Lett. 8, 4151 (2008).

${ }^{7}$ J. A. Lee, M. K. Shin, S. H. Kim, H. U. Cho, G. M. Spinks, G. G. Wallace, M. D. Lima, X. Lepró, M. E. Kozlov, R. H. Baughman, and S. J. Kim, Nat. Commun. 4, 1970 (2013).

${ }^{8}$ M. Barbaro, A. Caboni, P. Cosseddu, G. Mattana, and A. Bonfiglio, IEEE Trans. Inf. Technol. Biomed. 14, 758 (2010).

${ }^{9}$ J. S. Andrew and D. R. Clarke, Langmuir 24, 670 (2008).

${ }^{10}$ D. Isakov, E. de Matos Gomes, M. Belsley, B. Almeida, and N. Cerca, Nanoscale 4, 4978 (2012).

${ }^{11}$ D. Tu, S. Pagliara, A. Camposeo, L. Persano, R. Cingolani, and D. Pisignano, Nanoscale 2, 2217 (2010).

${ }^{12}$ L. Matlock Colangelo and A. J. Baeumner, Lab Chip 12, 2612 (2012).

${ }^{13}$ L. Persano, A. Camposeo, C. Tekmen, and D. Pisignano, Macromol. Mater. Eng. 298, 504 (2013).

${ }^{14}$ D. Isakov, E. de Matos Gomes, L. Vieira, T. Dekola, M. S. Belsley, and B. G. Almeida, ACS Nano 5, 73 (2011).

${ }^{15}$ M. Richard Lacroix and C. Pellerin, Macromolecules 46, 9473 (2013).

${ }^{16}$ A. Camposeo, F. Di Benedetto, R. Cingolani, and D. Pisignano, Appl. Phys. Lett. 94, 043109 (2009).

${ }^{17}$ D. Isakov, E. de Matos Gomes, I. Bdikin, B. Almeida, M. Belsley, M. Costa, V. Rodrigues, and A. Heredia, Cryst. Growth Des. 11, 4288 (2011).

${ }^{18}$ G. Singh, A. Bittner, S. Loscher, N. Malinowski, and K. Kern, Adv. Mater. 20, 2332 (2008).

${ }^{19}$ S. L. Sampson, L. Saraiva, K. Gustafsson, S. N. Jayasinghe, and B. D. Robertson, Small 10(1), 7882 (2014).

${ }^{20}$ C. E. Chang, V. H. Tran, J. B. Wang, Y. K. Fuh, and L. W. Lin, Nano Lett. 10, 726 (2010).

${ }^{21}$ X. Chen, S. Y. Xu, N. Yao and Y. Shi, Nano Lett. 10, 2133 (2010).

${ }^{22}$ L. Persano, C. Dagdeviren, Y. Su, Y. Zhang, S. Girardo, D. Pisignano, Y. Huang, and J. A. Rogers, Nat. Commun. 4, 1633 (2013).

${ }^{23}$ M. Szafrański, A. Katrusiak, and G. J. McIntyre, Phys. Rev. Lett. 89, 215507 (2002).

${ }^{24}$ K. E. Strawhecker and E. Manias, Macromolecules 34, 8475 (2001).

${ }^{25}$ V. I. Kovalenko, A. A. Akhmadiyarov, A. E. Vandyukov, and A. R. Khamatgalimov, J. Mol. Struct. 1028, 134 (2012).

${ }^{26}$ S. E. Kichanov, D. P. Kozlenko, J. Wasicki, L. S. Dubrovinsky, P. Czarnecki, W. Nawrocik, B. N. Savenko, D. K. Pogoreliy, and K. M. Podurets, J. Mol. Struct. 921, 68 (2009).

${ }^{27}$ S. Jen, N. A. Clark, P. S. Pershan, and E. B. Priestley, J. Chem. Phys. 66, 4635 (1977)

${ }^{28}$ N. Balke, I. K. Bdikin, S. V. Kalinin, and A. L. Kholkin, J. Am. Ceram. Soc. 92, 1629 (2009).

${ }^{29}$ V. Sencadas, C. Ribeiro, I. K. Bdikin, A. L. Kholkin, and S. Lanceros Mendes, Phys. Status Solidi A 209, 2605 (2012).

${ }^{30}$ R. Gonçalves and A. L. Kholkin, Energy Harvesting for Smart Miniaturized Systems in Energy Harvesting with Piezoelectric and Pyroelectric Materials, edited by N. Muensit (Trans Tech Publications, 2010).

${ }^{31}$ I. Lubomirsky and O. Stafsudd, Rev. Sci. Instrum. 83, 051101 (2012).

${ }^{32}$ K. T. Chung, B. A. Newman, and J. I. Scheinbeim, J. Appl. Phys. 53, 6557 (1982).

${ }^{33}$ Y. Yang, J. H. Jung, B. K. Yun, F. Zhang, K. C. Pradel, W. Guo, and Z. L. Wang, Adv. Mater. 24, 5357 (2012). 